Proc. Indian Acad. Sci., Vol. 87 A (E \& P Sciences-4), No. 11, November 1978, pp. 245-254, (C) printed in India

\title{
In situ magnetic measurements on igneous rock bodies*
}

\author{
C RADHAKRISHNAMURTY, S D LIKHITE and \\ P W SAHASRABUDHE $\dagger$ \\ Tata Institute of Fundamental Research, Bombay 400005 \\ $\dagger$ Physics Department, University of Zambia, Lusaka, Zambia
}

MS received 3 July 1978; revised 4 September 1978

\begin{abstract}
A simple method for an in situ measurement of susceptibility and low-field hysteresis on rock bodies like dykes and flows is described. Extensive use of this method indicates that in some dykes and flows, the nature of the magnetic grains varies rapidly across the bodies. Making use of such observations, it seems possible to select stable rock samples for palaeomagnetic work and also understand to some extent the origin of remanent magnetization in different types of rock bodies.
\end{abstract}

Keywords. Susceptibility: hysteresis; magnetic grains: igneous rock bodies.

\section{Introduction}

Several types of investigations were made on samples of a single lava (Watkins and Heggarty 1965; Ade-Hall et al 1968; Lawley and Ade-Hall 1971; Parry 1974), or a dyke (Smith and Prevot 1977), pillow basalt (Watkins et al 1970) or columnar basalt (Radhakrishnamurty et al 1971), and suites of basalts (Ade-Hall et al 1965; Like 1966; Soffel 1975; Radhakrishnamurty et al 1977), to understand the magnetic properties of the rocks in terms of the development of the iron oxide minerals in them. However, only a few of these studies were made on the samples taken out at some known spacings in the rocks bodies with a view to relate the properties to petrogenesis and alteration.

In the case of a single lava or a dyke, the samples that could be taken out for an investigation has to be limited to a reasonable number and it is but natural that any variations in magnetic properties over short intervals like a few centimeters could be missed easily. Hence, it would be helpful to have and use techniques that would permit in situ measurement more or less continuously on a rock body, to detect short range variations, if any.

An instrument for the in situ measurement of susceptibility and low-field hysteresis has been developed and the results of its use on some dykes are described.

\section{Susceptibility and low-field hysteresis apparatus}

The basic principles of operation of the apparatus are the same as those described

*Presented at the IAGA/IAMAP Joint Assembly session on 'rock magnetism;' Seattle, Washington, August 1977 
earlier (Likhite and Radhakrishnamurty 1965) but the new unit has been reduced in size and works on a battery pack. Figure 1 shows the photograph of the apparatus as it is used for probing a hand sample. The block diagram and the detailed circuit diagrams are given in figures $2 a$ and $2 b$ respectively.

For continuous measurement on a rock surface, the coil system is moved centimetre by centimetre and the hysteresis loop or a signal proportional to the susceptibility of the small area of the rock body beneath the pick-up coil is observed. As in other electrical or electromagnetic probing methods, it can be presumed that a hemispherical sample of the rock having nearly the same diameter of the pick-up coil contributes to the signal.

The special feature of the coil system is that although the pick-up coil occupies a considerable portion of the centre of the Helmholtz coil thereby sensing a nonuniform alternating magnetic field, it could be balanced to nearly the same degree as in a conventional laboratory system. This condition could be achieved by a thin winding section of the pick-up coil and a well matched circuitry. The apparatus could thus be used for probing the rock surface or on hand samples or rock chips. Although a portable oscilloscope is sufficient to carry out the study of magnetic variation, a miniscope-digital multimeter like the Tectronix 213 Miniscope/DMM could offer more reading accuracy and flexibility.

\section{In situ magnetic study on dykes}

Using the apparatus described above, the magnetic variations in several dykes of the Deccan trap region have been investigated. Some dykes showed only variation of susceptibility without any Rayleigh loops and others showed different types of Rayleigh loops as well across the width of the dyke.

One very surprising result is that totally constricted Rayleigh loop was observed only for a few centimeter wide region of a $200 \mathrm{~cm}$ wide dyke. In fact, two thin strips showing such a property have been found to run parallel to and at about $30 \mathrm{~cm}$ from either edge of the dyke. It is from these dykes showing variation in susceptibility and Rayleigh loops that an insight into the development of the magnetic grains in the rock bodies could be sought.

\section{Nature of the magnetic grains in dyke rocks}

Samples showing different types of Rayleigh loops were collected from the dyke and their thermomagnetic and hysteresis properties were studied in the laboratory. Figure $3 a$ shows the sampling locations. The susceptibility-temperature $(k-T)$ curves for the samples are shown in figure $3 b$. The Rayleigh loops obtained for the samples of the regions 1.2 and 3 are given in figure $3 \mathrm{c}$; and the high field hysteresis loops for the same samples at $25^{\circ} \mathrm{C}$ and $-196^{\circ} \mathrm{C}$ are shown in figure $3 \mathrm{~d}$ and $3 \mathrm{e}$.

Rayleigh loops shown by the samples indicate the presence of interacting superparamagnetic (SP) particles (Radhakrishnamurty and Sastry 1970; Neel 1970). However, from the type of the Rayleigh loop and the high field hysteresis properties (Radhakrishnamurty and Deutsch 1974) it can be inferred that all the three samples (figure 3) contain some amounts of single-domain (SD) and cation deficient (CD) 


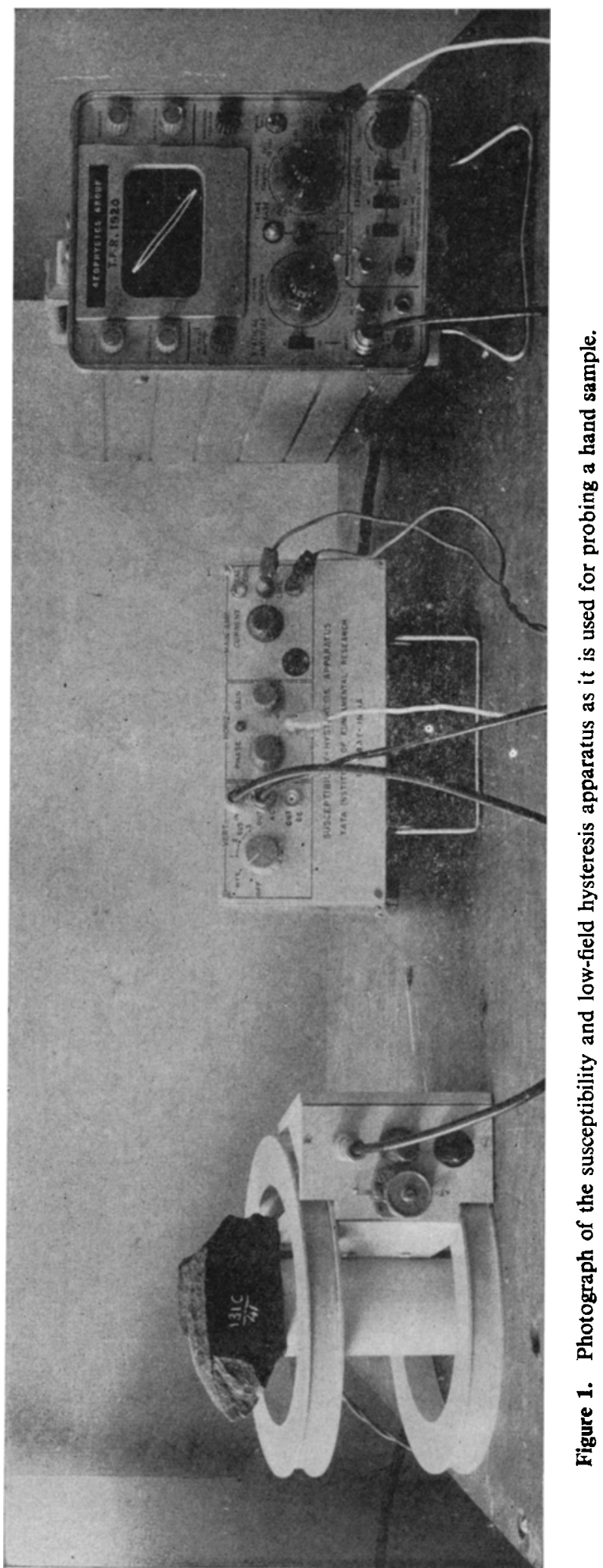




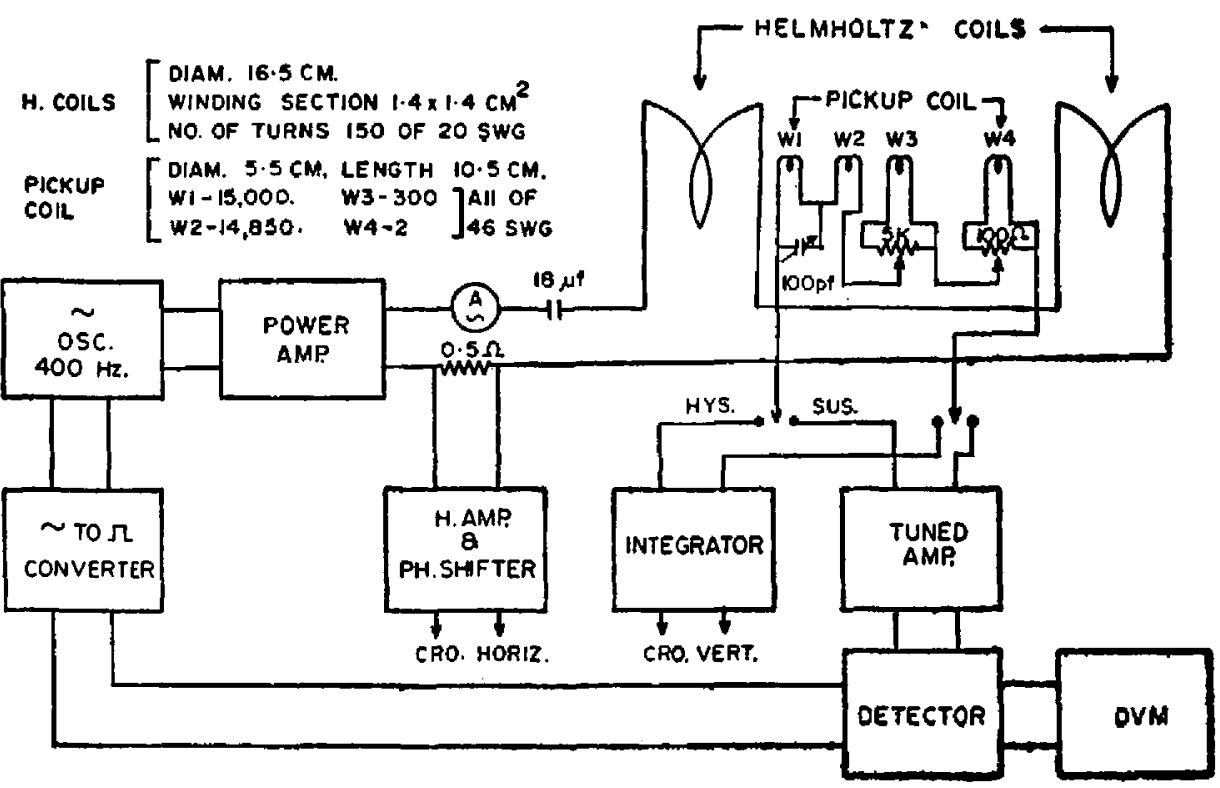

Figure 2a. Block diagram of the apparatus.
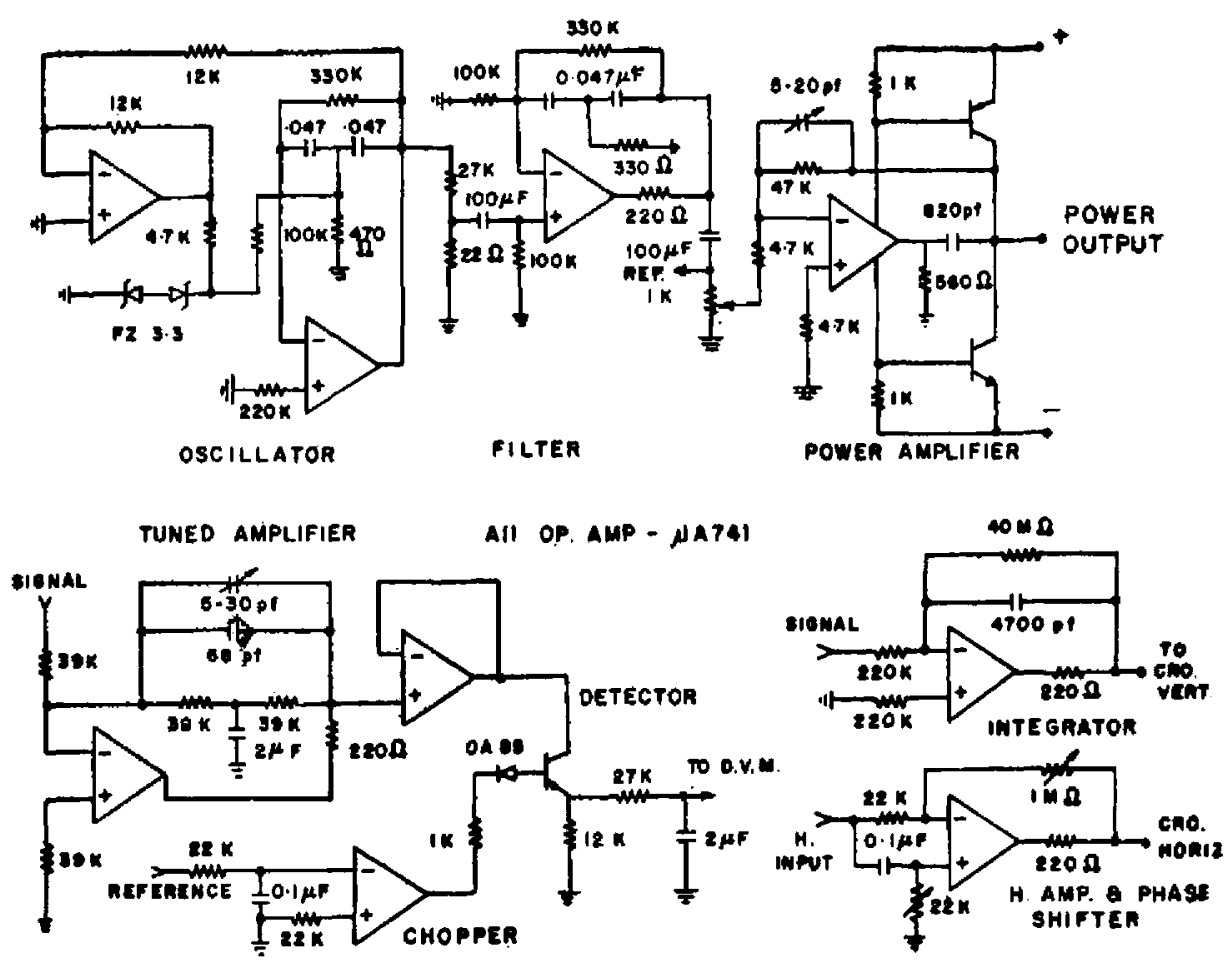

Figure 2b. Detailed circuit diagrams of the apparatus. 

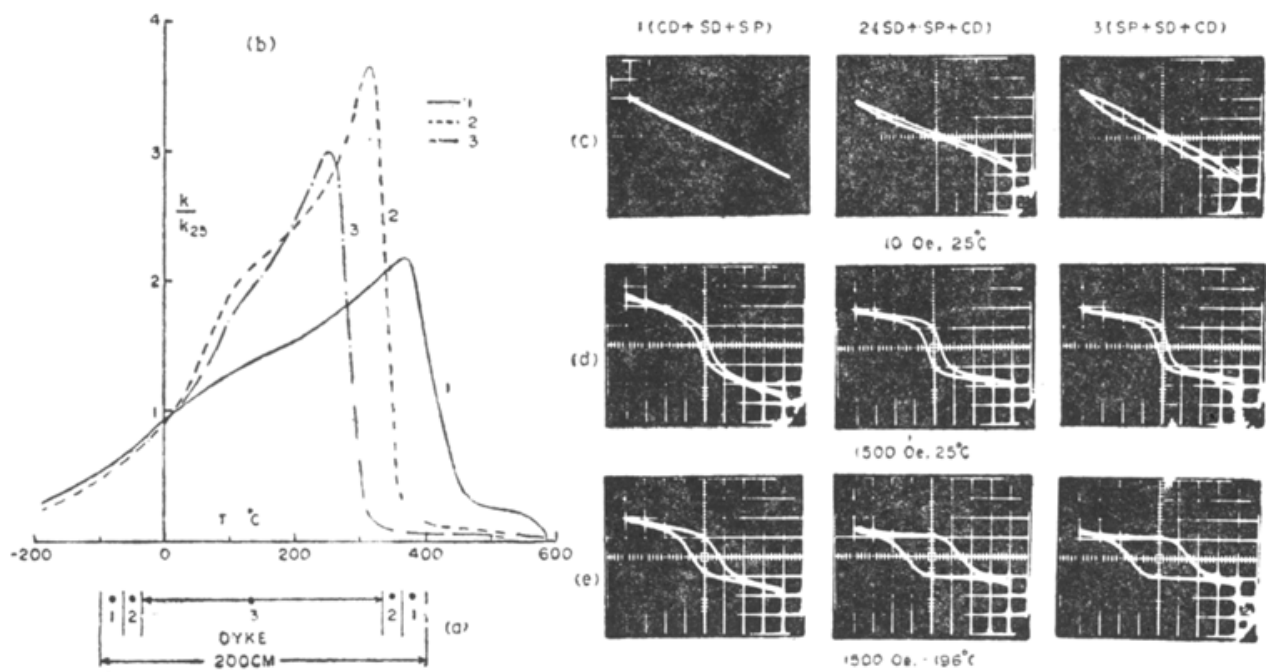

$1000,25^{\circ} \mathrm{C}$
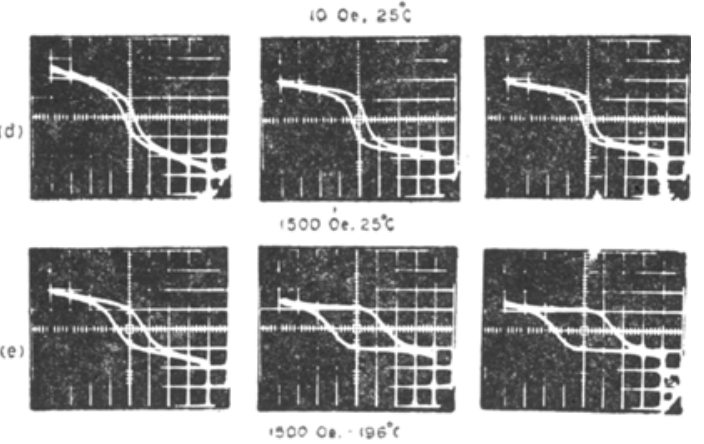

Figure 3. Low-field thermomagnetic curves and hysteresis loops at different fields and temperatures for the dyke samples.

magnetic grains as well, though the proportion of each of these grains may differ in different samples. Thus, the progressive increase in the coercive force $\left(H_{c}\right)$ and the value of relative remanence ( $R$-ratio of remanent-to-maximum intensity) from sample 1 to 3 at $-196^{\circ} \mathrm{C}$ (figure $3 \mathrm{c}$ ) compared to their respective values at $25^{\circ} \mathrm{C}$ (figure $3 \mathrm{~d}$ ), indicate the proportionate increase in the SD and SP particles. The values of $H_{c}$ and $R$ at $-196^{\circ} \mathrm{C}$ for sample 1 are lower than those of samples 2 and 3 because of the relatively more abundance of $\mathrm{CD}$ grains whose characteristic feature is to decrease these values at low temperatures (Radhakrishnamurty et al 1971).

Though, an approximate estimate of the relative proportion of the various types of the grains in each of these samples could be made on the basis of the criteria proposed earlier (Radhakrishnamurty et al 1977), a qualitative specification may be sufficient for the present discussion. Thus, the sequence of the grain types given for each of the three samples (figure 3 at the top) may be considered to represent decreasing proportion of their reiative abundance in each sample.

Figure 4 shows the heating and cooling curves for the different samples. It is noteworthy that all the heating curves have nearly the same shape though the steepest fall in the susceptibility takes place at different temperatures for these samples. However, there is a drastic change in the shapes of the curves on the cooling cycle. For sample 1 the CD content increased on heating while the SP content fell below the detectable limit as it did not show even a thin Rayleigh loop after cooling. Sample 2 may be said to maintain its contents with a minor redistribution of the grain states and with the formation of some additional magnetic material as indicated from the increase in intensity. In sample 3 , the $\mathrm{CD}$ content fell below the detectable limit while the relative proportion of SP increased, as this sample showed a much wider Rayleigh loop on cooling.

When SP or SD grains change to CD state, grain growth and some increase in the intensity of magnetization are to be expected, while in the reverse process, if and when takes place, some reduction both in grain size and intensity could be expected, 


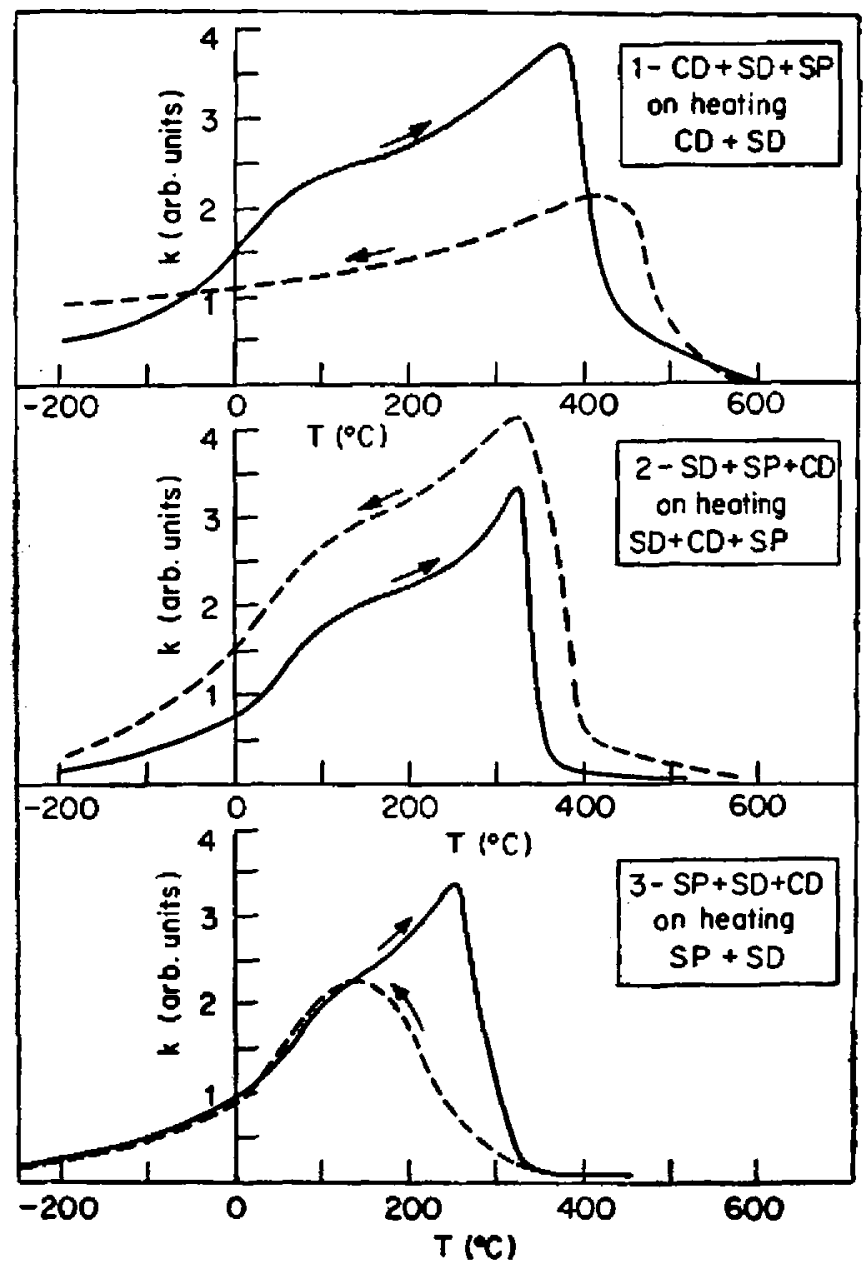

Figure 4. Thermomagnetic curves showing the heating and cooling cycles for the dyke samples.

assuming no other magnetic material formation from the glassy matrix of a basaltic sample on heating. However, the changes that may take place on heating basalts are unpredictable. Nevertheless, it seems possible to explain the magnetic properties of virgin and heat-treated samples of basalts in terms of the different grain states from their characteristic features.

Alternatively, the steep fall in the value of susceptibility shown by different samples (figure $3 \mathrm{~b}$ ) may be presumed to result from the presence of large titanomagnetite grains with different amounts of titanium in solid solution and showing corresponding Curie points. On heating the samples, the titanium either exsolved in some way to increase the $T_{c}$ as in the case of samples 1 and 2 , or some excess titanium present in the sample diffused in, resulting in a decrease in $T_{c}$ as for the case of sample 3 (see figure 4). Such a dual mechanism was proposed by Soffel (1975) to account for the changes he observed on heating some volcanic rocks. But then, the low and high field hysteresis properties observed for these dyke samples (figure 3) will have to 
remain shrouded in a mystery as no explanation seems to be possible for these properties on the basis of large titanomagnetite grains.

On the other hand, the earlier model, based on the mixtures of different states of magnetite grains without involving titanomagnetites, could explain consistently all the observed properties, including the hysteresis loops, of these dykes samples, as discussed above. However, in this case the effective magnetic grain size has to be presumed to be much smaller than the physical size of the iron oxide grains present in the sample, the justification of which is discussed in the next section.

\section{Development of the magnetic grains in the dykes}

Optical observations on polished sections of all the dyke samples revealed the presence of skeletal crystals of the opaque mineral. Although, some increase in the physical size of the opaque grains could be noticed in the samples taken from edge to the middle of the dyke, it is difficult to compute any meaningful size for the grains because of the skeletal nature. Chemical analysis of the separated iron oxide grains (impure because of the skeletal nature) showed the presence of considerable amounts of titanium in them to be able to form low $T_{c}\left(\sim 100^{\circ} \mathrm{C}\right)$ titanomagnetites. But, in any case the magnetic properties indicating the presence of SP particles in the samples could not be understood in terms of the observable opaque grains. Also, it is important to note that while the physical size of opaque grains is definitely larger in the samples towards the center of the dyke, the observations of Rayleigh loops indicate more abundance of SP particles in these samples, which is an apparent contradiction.

However, the above contradiction could be resolved and a probable mechanism for the development of different magnetic states of the grains in the dyke samples could be conceived by showing due consideration for the chemical composition and the physical size of the opaque grains. Only it has to be presumed that the effective magnetic grain size is small so that small regions of a large grain could behave like SP or SD particles in these dyke samples. For all these dyke samples, the steepest fall of the susceptibility at different temperatures (figure 3a) is presumed to be related to the effective magnetic grain size and interaction effects among them and will not represent a phase with a corresponding $T_{c}$. The observation of different thermomagnetic curves for synthetic magnetite (and titanomagnetite) grains of different sizes by Day (1975) could be considered to support the virtual nature of any $T_{c}$ if grain size effects are present. It may be pointed out that only in the case of a sample containing large multidomain (MD) grains of a magnetic material, could the $T_{c}$ be determined without any ambiguity. The absence of multidomain grains of titanomagnetites in basalts has been disscussed in detail elsewhere (Radhakrishnamurty et al 1978).

Normally, in any rock sample some of its SP or SD particles could get oxidised to CD state and these CD grains could still be small enough to behave like single domains. For example, as pointed out in $\S 4$, the chilled edges of the dyke retain considerable amounts of gases and because of their presence more of the iron oxide grains in this region could get oxidized to CD state compared to those in the middle of the dyke.

Hence, the explanation of the observed magnetic properties of the dyke samples on grain size and state effects seems to be quite consistent. However, because of the 
experimental difficulties neither the exact size of the effective magnetic grain nor their composition could be determined directly.

It is very likely that the magnetic states of the iron oxide grains in different types of igneous rock bodies might have formed soon after the solidification of the magma. Some insight on this aspect could be obtained by studying the uniformity or otherwise of the magnetic properties of a rock body in relation to its physical size and shape. As for the dyke under consideration, the magnetic properties have been found to be uniform and symmetrical with respect to the axis of the dyke. It may then be considered that the stable remanent magnetization believed to be due to the SD and CD grains, may be of primary origin. However, it may be possible that the original iron oxide grains in such rock bodies were of 'true' MD titanomagnetite but now only its composition can be determined and not its original magnetic state. The titanomagnetite grains could have been subdivided magnetically later on due to some yet unknown process, imparting different magnetic properties observable now. In this case original domain state of the titanomagnetite grains and the subsequent changes both in time and nature would be difficult to deduce. Thus, further investigations would be required to deduce the complicated history of the iron oxide grains in basalts.

The apparatus and the in situ measurement procedure described above are also useful in selecting samples for palaeomagnetic work. Samples may be preferably collected from outcrops that do not show any Rayleigh loops, which implies the absence of SP particles to any major extent. If a dyke or lava outcrop shows Rayleigh loops, samples may be taken out from those regions which show the thinnest loops, as such samples could be expected to contain a minimum proportion of SP particles among the grain states present.

\section{Conclusion}

The in situ magnetic measurement procedure might be helpful in selecting samples for paleomagnetic purposes and to understand the nature and development of magnetic grains in igneous rock bodies.

\section{Acknowledgements}

We thank Professor R L Wilson, University of Liverpool, U K and Professors E R Deutsch and G S Murthy, Memorial University of Newfoundland, for their valuable comments on this manuscript.

\section{References}

Ade-Hall J M, Wilson R L and Smith P J 1965 Earth Planet. Sci. Lett. 9323

Ade-Hall J M, Khan M A and Wilson R L 1968 Geophys. J. 16375

Day Ron 1975 Earth Planet. Sci. Lett. 2795

Lawley E A and Ade-Hall J M 1971 Earth Planet. Sci. Lett. 11113

Like C B 1966 Pure Appl. Geophys. 65132

Likhite S D and Radhakrishnamurty C 1965 Bull. NGRI. 31 
Neel L 1970 C. R. Acad. Sci. Paris 2701125

Parry L G 1974 Geophys. J. 38225

Radhakrishnamurty C and Deutsch E R 1974 J. Geophys. 40453

Radhakrishnamurty C, Likhite S D, Raja P K S and Sahasrabudhe P W 1971 Nature (London) Phys. Sci. 23533

Radhakrishnamurty C, Likhite S D, Deutsch E R and Murthy G S 1978 (in preparation)

Radhakrishnamurty C, Likhite S D and Sahasrabudhe P W 1977 Phys. Earth Planet. Inter. 13289

Radhakrishnamurty C and Sastry N P 1970 Proc. Indian Acad. Sci. A72 94

Smith B M and Prevot M 1977 Phys. Earth Planet. Inter. 14120

Soffel H $1975 \mathrm{~J}$. Geophys. 41401

Watkins N D and Heggarty S E 1965 Nature (London) 206797

Watkins N D, Paster $\Upsilon$ and Ade-Hall J M 1970 Earth Planet. Sci. Lett. 8322 